Simone Lemos ${ }^{2}$

Davi Rumel ${ }^{3}$

\title{
Ocorrência de disfonia em professores de escolas públicas da rede municipal de en- sino de Criciúma-SC ${ }^{1}$
}

\author{
Occurrence of dysphonia among teachers \\ from Public Schools in Criciúma-SC
}

${ }^{1}$ Trabalho extraído da Dissertação de Mestrado de Simone Lemos, intitulada Ocorrência de disfonia em professores de escolas públicas da rede municipal de ensino de Criciúma - SC, defendida em 2004 na Universidade do Extremo Sul Catarinense.

${ }^{2}$ Mestra em Saúde Coletiva pela Universidade do Extremo Sul Catarinense (Unesc), fonoaudióloga da Prefeitura de Criciúma.

${ }^{3}$ Doutor em Epidemiologia pela Faculdade de Saúde Pública da Universidade de São Paulo (FSP-USP), diretor adjunto da Agência Nacional em Vigilância Sanitária (ANVISA).

\begin{abstract}
Resumo
Este estudo tem por objetivo a caracterização da ocorrência de disfonia em 236 professores da rede municipal de ensino de Criciúma e a determinação dos fatores a ela associados em decorrência do exercício profissional. Os professores responderam a um questionário sobre as características do local de trabalho, os hábitos vocais, a autopercepção da disfonia, a procura por especialista, o uso de medicamentos e os cuidados com a voz. Os professores que mencionaram três ou mais sintomas vocais passaram por uma triagem fonoaudiológica. Após a aplicação da triagem, procedeu-se a um exame otorrinolaringológico. Realizou-se um teste de associação pelo qui-quadrado para se verificar a associação entre as variáveis independentes e a presença de disfonia. Os resultados mostraram prevalência de disfonia na população total em estudo, a qual foi estimada entre $17,15 \%$ (partindo-se do pressuposto de que as perdas não têm disfonia) a 46,80\% (partindo-se do pressuposto de que as perdas têm disfonia) dos professores. Os fatores associados à disfonia foram o hábito de falar muito, a orientação sobre o uso da voz, a procura por especialista e a condição da voz em ex-fumantes. Este estudo visou também à demonstração de que, através de programas de ação preventiva, pode-se, com o empenho do poder público, evitar o agravamento e o aparecimento destas alterações.
\end{abstract}

Palavras-chaves: voz, disfonia, professores.

\begin{abstract}
Lemos S. Occurrence of dysphonia among teachers from Public Schools in Criciúma-SC. 2004. [Master thesis dissertation-University of Extreme South of Santa Catarina]. The purpose of this study is to figure out the prevalence and the identification of factors that are associated with dysfonia among 236 teachers from Public Schools in Criciuma-SC. The teachers answered a questionnaire about the characteristics of their work place, their vocal habits, self perception of dysphonia, search for professional help, the use of medicine, and about their voice care. The teachers who mentioned three or more vocal symptoms went through a vocal screening. After being screened, the teachers were sent to an otolaryngological evaluation. A chi-square test was made in order to detect association among independent variables and dysphonia. The result showed a range between 17,15\% (considering lost of follow-up as non dysphonia) to $46,80 \%$ (considering lost of follow-up as dysphonia) of dysphonia prevalence among teachers. The associated factors for dysphonia were the habit of speaking too much, the amount of information on voice good practices, search for expertise help and being an ex-smoker. This study also aimed at pointing at the importance of preventive actions that could be taken with governmental support to prevent this kind of health problems.
\end{abstract}

Keywords: voice, dysphonia, teachers. 


\section{Introdução}

A atividade exercida pelos professores é considerada de risco quanto ao desenvolvimento de alterações vocais. Essas alterações muitas vezes se agravam com o mau uso ou o abuso do mecanismo fonatório, podendo estar associadas a diversos fatores, tais como o uso de fumo, a acústica inadequada, a presença de poeira, a carga horária excessiva (PORDEUS, PALMEIRA \& PINTO, 1996).

A disfonia é definida como a alteração vocal caracterizada pela presença de som sugestivo de problemas em nível de fonte glótica, ou seja, quando a qualidade vocal se mostra alterada na análise percetivo-auditiva (DRAGONE, 1996). Também Boone \& Mac Farlane (1994) consideram disfonia qualquer problema na vocalização normal.

Muitas vezes, o impacto da disfonia é devastador, pois pode gerar limitações vo-

\section{Literatura}

Encontram-se na literatura muitas referências sobre a voz do professor com estudos que abordam o surgimento e o desenvolvimento das alterações vocais e sua possível associação com as condições de trabalho.

Fernandes (1996) constatou que, de um total de 92 professores, 34\% apresentavam queixa de alteração vocal. Scalco, Pimentel \& Pilz (1996) constataram que entre as 50 professoras pesquisadas, 46\% apresentaram alteração vocal acusticamente perceptível.

Simões (2001) constatou, mediante triagem vocal, que 74 professoras apresentaram qualidade vocal alterada, o que representa uma prevalência de disfonia de $79 \%$. Ao analisarem-se as associações entre a disfonia e os principais fatores de risco para esse agravamento, concluiu-se que não houve associação estatisticamente significativa entre presença de disfonia e idade, estado civil, escolaridade ou tempo de serviço. Com relação às características físicas do local de trabalho, não houve associação entre a presença de disfonia e a acústica da sala, eco na sala, ruído, fumaça, umidade, temperatura, iluminação, limpeza, uso de produtos químicos irritativos e tamanho da sala. Porém, a presença cais além de incapacitar o professor (RODRIGUES, AZEVEDO \& BEHLAU, 1996). As licenças médicas prolongadas por disfonia são registradas como enfermidade profissional, devendo, portanto, serem investigadas as suas causas (BRUNETTO et al., 1986).

A disfonia vem sendo discutida como doença ocupacional desde 1997, através de seminários, por uma equipe de profissionais, dentre os quais fonoaudiólogos, médicos do trabalho e otorrinolaringologistas (VIOLA et al., 1997).

Ainda não há na formação curricular do professor uma disciplina com o objetivo de preservar sua voz, buscando seu melhor desempenho. Sem esse conhecimento, o professor incorre infalivelmente em mau uso ou uso abusivo da voz frente às inúmeras tarefas que lhe são exigidas no seu cotidiano (DRAGONE et al., 1999).

de poeira foi estatisticamente associada à disfonia.

Neto et al. (2000) ressaltaram que as características de trabalho mais fortemente associadas às queixas de doenças foram: salas inadequadas, trabalho repetitivo, exposição à pó de giz, ambiente intranqüilo e estressante, ritmo acelerado de trabalho, desempenho das atividades sem materiais e equipamentos adequados e posição de trabalho incômoda.

Oliveira et al. (1995) comentaram a presença de poeira no ambiente de trabalho e também o ruído externo como causas de interferência no decorrer das aulas. Garcia, Torres \& Shasat (1986) apontaram diversos hábitos tóxicos que atuam como irritadores, além de fatores que incidem negativamente sobre a laringe, tais como fumo, álcool, mudanças de temperatura, entre outros. Penteado, Teixeira \& Bicudo (1999) estabeleceram em seus estudos a relação entre saúde vocal/distúrbios da voz e as condições de trabalho e a vida do professor. Polizzi, Barría \& Campos (1986) constataram que, dos 44 professores pesquisados, 27 (61\%) apresentaram disfonia, tendo 29 tirado licença médica nos últimos três anos devido a problemas de voz. Ferreira et al. (2003) definiram a acústica da sala de aula 
insatisfatória, a referência de local ruidoso, o tamanho da sala inapropriado e a tempe-

\section{Material e Método}

O delineamento do estudo é do tipo transversal. Participaram do presente estudo 236 professores das 13 escolas de Educação Infantil e do Ensino Fundamental da rede municipal de ensino da cidade de Criciúma-SC mediante relatório fornecido pela Secretaria de Educação. A amostra é de conveniência, sorteada de acordo com o critério de facilidade de locomoção, tendo-se em vista a necessidade de ir várias vezes às escolas. A coleta de dados foi feita através de entrevista, estruturada com aplicação de questionário, triagem fonoaudiológica e exame físico com o médico

\section{Resultados e Discussão}

Dos 236 professores, 217 (91,95\%) são do sexo feminino e 19 (8,15\%), do sexo masculino. A idade mediana é de 37 anos, com mínima de 21 anos e máxima de 60 anos. Cerca de 124 (52,55\%) professores trabalham nesta profissão há mais de 10 anos e 120 (50,9\%) trabalham com uma carga horária de 25 a 40 horas semanais. Identificou-se que $160(67,8 \%)$ professores mencionaram que o número de alunos por turma varia de 20 a 30. Do total de professores pesquisados, 190 (80,50\%) relataram problemas de voz cujos sintomas vocais mais citados foram: rouquidão - 154 (81\%); cansaço ao falar - 138 (72,6\%); ardência 110 (57,9\%); tosse - 102 (53,6\%); perda da voz - 100 (52,6\%); e pigarro - 92 (48,5\%). Dos 190 professores que alegaram problemas vocais, 116 (61,05\%) apresentavam esses sintomas há mais de 3 anos; 6 (15,25\%) já faltaram ao trabalho devido a problemas com a voz; 150 (79,1\%) alegaram que falam muito; 124 (65,2\%), que falam alto; $72(37,8 \%)$, que fazem esforço ao falar; 68 $(35,8 \%)$, que poupam a voz; e $38(20,40 \%)$, que gritam. Dos 236 professores, 135 $(57,2 \%)$ mencionaram que a acústica da sala de aula não é satisfatória; 126 (53,4\%), que o local é ruidoso; 153 (64,85\%) mencionaram desconforto com o pó de giz durante as aulas; 162 (68,65\%) não receberam orientação sobre o uso da voz; e 38 (16,1\%) apresentaram o hábito de fumar.

Não se encontrou associação estatisticamente significativa entre presença de disfo- ratura do ambiente como fatores de risco para o surgimento de alterações vocais. otorrinolaringologista para os casos encaminhados. Os dados foram organizados em um gerenciador de banco de dados e posteriormente processados e analisados em um programa específico de Epidemiologia e Estatística (EPI INFO, versão 6.0). As variáveis foram analisadas de forma descritiva por meio de proporções. Realizou-se o teste do qui-quadrado para se determinar a associação entre as variáveis independentes e a presença de disfonia. Reportou-se a probabilidade de erro alfa. Consideraramse associações significativas aquelas com $\mathrm{p}<0,05$. nia e tempo de magistério, assim como nos estudos de Brunetto et al. (1986), Pordeus, Palmeira \& Pinto (1996), Russel, Oates \& Grenwood (1998) e Simões (2001).

Não houve associação estatisticamente significativa entre presença de disfonia e carga horária, semelhantemente aos achados de Urrutikoetxea, Ispizua e Matellanes (1995), Smith et al. (1998), Bacha et al. (1999) e Simões (2001).

Não se encontrou associação estatisticamente significativa entre presença de disfonia e número de alunos por turma nem entre presença de disfonia e nível de ensino, semelhantemente aos dados encontrados nos estudos de Simões (2001).

Quanto às características físicas do local de trabalho, não se encontrou associação estatisticamente significativa entre disfonia e acústica da sala, ruído e pó de giz, semelhantemente aos dados encontrados nos estudos de Simões (2001).

Não se verificou associação estatisticamente significativa entre presença de disfonia e uso de medicamentos nem entre presença de disfonia e faltas ao trabalho. Outros estudos não verificaram esta relação.

Houve associação estatisticamente significativa entre presença de disfonia e procura por especialista. Outros estudos não verificaram esta relação.

Não foi possível verificar associação estatisticamente significativa entre presença 
de disfonia e percepção de alteração vocal. Os resultados coincidem com os achados de Simões (2001) e Ferreira et al. (2003).

Encontrou-se associação estatisticamente significativa entre presença de disfonia e orientação sobre o uso da voz, o que era de se esperar, pois quem tem disfonia procura uma orientação sobre o uso da voz e quem não tem não o faz. Idealmente, todos deveriam procurá-la. Esta associação deveria ser não significante se os professores tivessem acesso a uma ação preventiva neste campo. Este resultado corroborou os estudos de Ferreira et al. (2003).

Tabela 1 Distribuição do número e da porcentagem de professores segundo as variáveis relativas à disfonia. Criciúma, 2004

\begin{tabular}{lccc}
\hline \multicolumn{1}{c}{ Variável } & Categoria & $n$ & Total \\
\hline Tempo de magistério & & 124 & 52,55 \\
Carga horária & 25 a 40 & 120 & 50,90 \\
Alunos por turma & 20 a 30 & 160 & 67,80 \\
Problema de voz & Sim & 190 & 80,50 \\
Apresenta sintoma & S anos & 116 & 61,05 \\
Falta ao trabalho & Sim & 6 & 15,25 \\
Acústica não satisfatória & Sim & 135 & 57,20 \\
Local ruidoso & Sim & 126 & 53,40 \\
Desconforto ao pó de giz & Sim & 153 & 64,85 \\
Orientação sobre uso da voz & Sim & 162 & 68,65 \\
Apresenta o hábito de fumar & Sim & 38 & 16,10 \\
\hline
\end{tabular}

Não se encontrou associação estatisticamente significativa entre presença de disfonia e fumo. Corroboraram os estudos de Brunetto et al. (1986) e Scalco, Pimentel \& Pilz (1996), Simões (2001) e Ferreira et al. (2003).

Foi possível verificar associação estatisticamente significativa entre a presença de disfonia e o fato de ser o professor ex-fumante. Todos os 25 professores ex-fumantes apresentaram disfonia. Talvez seja esta a razão que os levou a parar de fumar. Outros estudos não confirmaram esta relação.

Sobre os cuidados com a voz, não houve associação estatisticamente significativa entre a presença de disfonia e o hábito de beber água durante o uso da voz, poupar a voz, gritar, falar alto e fazer esforço ao falar. Esses resultados coincidem com os achados de Simões (2001).

Foi possível verificar associação estatisticamente significativa entre a presença de disfonia e o falar muito. Talvez esta seja uma exigência difícil de ser cumprida pelos professores. Os resultados coincidem com os achados de Ferreira et al. (2003).
A prevalência de disfonia na população total em estudo foi de $17,15 \%$ a $46,8 \%$. Estudos com professores brasileiros encontram uma porcentagem que varia de $30 \%$ a 79\%. Bacha et al. (1999) apresentaram ocorrência de disfonia em 30,9\% dos professores. Tenor et al. (2000) apresentaram $35 \%$. Dragone (1996) apresentou 50,6\%. Simões (2001), 79,6\%. Dragone et al. (1999) apresentaram 79,7\%. O fato de a prevalência ter sido inferior aos estudos encontrados na literatura justifica-se pelas perdas havidas durante a pesquisa. A triagem fonoaudiológica foi realizada em todos os professores que mencionaram três ou mais sintomas a partir do questionário aplicado. Dos 190 professores que mencionaram problemas vocais, foram triados apenas 77 , tendo sido os 113 restantes considerados normais. Esta medida foi adotada como critério de seleção embora não tenhamos encontrado na literatura referencial teórico que forneça dados sobre a quantidade de sintomas necessários para descrever-se um grupo que apresente distúrbios vocais ou seja predisponente aos mesmos. Porém, a prática fonoaudiológica confirma que três ou mais sintomas são indicativos 
de alteração vocal (PORDEUS, PALMEIRA \& PINTO, 1996). Se triados os 190 professores, essa prevalência subiria de 17,15\% para $46,8 \%$, o que ficaria bem próximo de alguns estudos nacionais. Poderíamos tam- bém atribuir os valores de prevalência encontrados ao fato de a triagem fonoaudiológica ser uma avaliação subjetiva e ter sido realizada por uma única fonoaudióloga: a própria pesquisadora.

Tabela 2 Teste de associação entre as variáveis relativas a características do trabalho, auto-percepção das características físicas do local de trabalho, uso de medicamentos, falta ao trabalho e procura por especialista, orientação recebida sobre uso da voz, percepção dos problemas de voz, estilo de vida e disfonia. Criciúma, 2004

\begin{tabular}{|c|c|c|c|c|}
\hline \multirow[b]{2}{*}{ Variável } & \multirow[b]{2}{*}{ Categoria } & \multicolumn{2}{|c|}{ Disfonia } & \multirow[b]{2}{*}{$\%$} \\
\hline & & $\underset{n}{\mathrm{Sim}}$ & $\begin{array}{c}\text { Não } \\
n\end{array}$ & \\
\hline \multirow{2}{*}{ Nível de ensino } & Educação Infantil & 3 & 5 & \multirow[b]{2}{*}{0,9500} \\
\hline & Ensino Fundamental & 33 & 36 & \\
\hline \multirow{3}{*}{ Tempo de magistério } & Até 5 anos & 6 & 4 & \multirow{3}{*}{0,4538} \\
\hline & 5 a 10 anos & 12 & 11 & \\
\hline & + de 10 anos & 18 & 26 & \\
\hline \multirow{3}{*}{ Carga horária } & - de $25 \mathrm{~h} / \mathrm{s}$ & 5 & 6 & \multirow{3}{*}{0,5213} \\
\hline & de 25 a $40 \mathrm{~h} / \mathrm{s}$ & 18 & 25 & \\
\hline & + de $40 \mathrm{~h} / \mathrm{s}$ & 13 & 10 & \\
\hline \multirow{3}{*}{ Alunos por turma } & - de 20 alunos & 0 & 3 & \multirow{3}{*}{0,3916} \\
\hline & de 20 a 30 & 24 & 26 & \\
\hline & + de 30 alunos & 12 & 12 & \\
\hline \multirow{2}{*}{ Acústica satisfatória } & Sim & 13 & 10 & \multirow{2}{*}{0,1605} \\
\hline & Não & 19 & 30 & \\
\hline \multirow{2}{*}{ Local ruidoso } & Sim & 20 & 27 & \multirow{2}{*}{0,6601} \\
\hline & Não & 12 & 13 & \\
\hline \multirow{2}{*}{ Desconforto a pó de giz } & Sim & 23 & 34 & \multirow{2}{*}{0,1760} \\
\hline & Não & 9 & 6 & \\
\hline \multirow{2}{*}{ Uso de medicamento } & Sim & 14 & 21 & \multirow{2}{*}{0,5228} \\
\hline & Não & 22 & 20 & \\
\hline \multirow{2}{*}{ Procura por especialista } & Sim & 7 & 18 & \multirow{2}{*}{ "0,0129 } \\
\hline & Não & 29 & 23 & \\
\hline \multirow{2}{*}{ Falta ao trabalho } & Sim & 7 & 15 & \multirow{2}{*}{0,1580} \\
\hline & Não & 24 & 24 & \\
\hline \multirow{2}{*}{ Orientação sobre uso da voz } & Recebeu & 6 & 21 & \multirow{2}{*}{ * 0,0026} \\
\hline & Não recebeu & 27 & 19 & \\
\hline \multirow{2}{*}{ Problemas de voz } & $\operatorname{Sim}$ & 35 & 39 & \multirow{2}{*}{0,1073} \\
\hline & Não & 1 & 2 & \\
\hline \multirow{2}{*}{ Fumo } & Sim & 5 & 4 & \multirow{2}{*}{0,4214} \\
\hline & Não & 26 & 37 & \\
\hline & Sim & 4 & 0 & \\
\hline Ex-fumante & Não & 21 & 37 & " 0,0125 \\
\hline
\end{tabular}

"Estatisticamente significativo 


\section{Conclusão}

Os dados revelaram que grande parte dos professores abordados neste estudo alegou falta de conhecimento com relação aos cuidados com a voz. A desinformação quanto aos cuidados a serem tomados é uma realidade desta categoria profissional, mas não um fator determinante. "Acreditase que isso decorra de alguns fatores como a deficiência dos cursos de formação dos professores, a falta de atenção da comunidade escolar e a insuficiente atuação preventiva." (SCALCO, PIMENTEL \& PILZ, 1996, p. 26).

Com base na análise dos dados obtidos neste estudo, é possível concluir-se o quanto é fundamental a implantação de propostas preventivas de atuação na atenção à saúde vocal do professor em programas de saúde escolar ou de saúde do trabalhador e a realização de diagnóstico precoce visando à prevenção e evitando-se assim que o professor adoeça.

Identificou-se um número significativo de professores que auto-referem transtornos vocais. Este achado demonstra o quanto os professores estão atentos ao uso da voz.

Dos professores que têm sintomas, a maioria alegou que os apresenta há mais de três anos. Portanto, parece que estes profissionais estão convivendo já há bastante tempo com problemas vocais e que as queixas com relação à voz aparecem bem antes das alterações vocais propriamente ditas. Talvez, com o passar do tempo, eles estejam se adaptando à qualidade vocal alterada.

A disfonia tem impacto no trabalho, pois, dos 236 professores, 15,25\% já faltaram ao trabalho devido a problemas de voz. Aparentemente, eles faltam apenas o

\section{Referências Bibliográficas}

BACHA, S. M. C. et al. Incidência de disfonia em professores de pré-escola do ensino regular da rede particular de Campo Grande/MS. Revista Pró-Fono, v. 11, n. 2, p. 8-15, 1999.

BOONE, D. R.; \& MAC FARLANE, S. C. $A$ voz e a terapia vocal. Porto Alegre: Artes Médicas, 1994.

BRUNETTO, B. et al. Mitos y realidades de la disfonia profisional. Rev. Otorrinolaringologia. 46, p. 115-120, 1986. suficiente para que a voz volte e lhes dê as mínimas condições para o trabalho, independentemente de estarem totalmente recuperados. $\mathrm{Na}$ verdade, os professores têm medo de perder seu emprego, receiam a limitação do exercício profissional e as conseqüentes implicações na continuidade de suas tarefas.

A não-significância estatística com relação à disfonia e às características físicas do local de trabalho no desenho deste estudo devem-se talvez ao fato de ter sido avaliada a "percepção do professor" frente à sua atividade de trabalho. Isso, porém, não diminui a magnitude do problema.

Seria relevante a condução de estudos longitudinais que pudessem estabelecer com maior validade a associação entre as características do ambiente do trabalho e o processo de enfermidade dos professores, uma vez que este seria o ideal e principal nível de atuação preventiva.

Não se pode negligenciar o fato de que os resultados das variáveis relacionadas às características do local de trabalho expressam uma condição de trabalho intenso, podendo-se afirmar que, entre os professores que não apresentam disfonia, há fatores de proteção que lhes favorecem. Provavelmente tais fatores estão ligados às estratégias individuais e coletivas desenvolvidas por eles.

Os resultados deste estudo revelaram que no município de Criciúma não há um serviço realmente comprometido com os interesses dessa população e ressaltaram a necessidade de medidas que englobem diferentes níveis de prevenção a fim de propiciar-se uma maior proteção e uma assessoria a estes profissionais.

DRAGONE, M. L. S. Ocorrência de disfonias em professores: fatores relacionados à voz profissional. 1996. Monografia (Especialização) - Centro de Estudos da Voz, São Paulo, 1996.

DRAGONE, M. L. S. et al. Desgaste vocal do professor: um estudo longitudinal. Rev. Soc. Bras. Fonoaudiol., v. 3, n. 5, p. 50-57, 1999.

FERNANDES, C. R. J. Caracterização de um grupo de professores com alteração vocal 
da pré-escola do município do Taboão da Serra/SP. 142f. 1996. Dissertação (Mestrado) - Pontifícia Universidade Católica de São Paulo, São Paulo, 1996.

FERREIRA, L. P. et al. Condições de produção vocal de professores da prefeitura do município de São Paulo. Revista Distúrbio da Comunicação. v. 14, n. 2, p. 275-310, 2003.

GARCIA, C. O. TORRES, R. P; \& SHASAT, A. D. D. Disfonías ocupacionales: estudio de 70 casos. Rev. Cub. Med. v. 25, n. 10, p. 998-1009, 1986.

NETO, S. M. S. et al. Condições de trabalho e saúde de professores da rede particular de ensino. Revista Baiana de Saúde Públi$c a$, v. 24, n. 1-2, p. 42-56, 2000.

OLIVEIRA, I. B. et al. Distúrbios vocais em professores de pré-escola e primeiro grau. In: FERREIRA, L. P. et al. Voz profissional: o profissional da voz. Carapicuíba: Pró-Fono, 1995. p. 173-179.

POLIZZI, J. A.; BARRÍA, M. A.; \& CAMPOS, A. Disfonia funcional y evaluacion fonoaudiologica de un grupo de docentes universitarios. Rev. Otorrinolorigologia. 46, p. 81-84, 1986.

PENTEADO, R. Z.; TEIXEIRA, I. M.; \& PEREIRA, B. A voz do professor: relações entre trabalho, saúde e qualidade de vida. Rev. bras. saúde ocup. v. 95-96, n. 25, p. 109-130, 1999.

PORDEUS, A. M. J.; PALMEIRA, C. T.; \& PINTO, V. C. V. Inquérito de prevalência de problemas da voz em professores da Universidade de Fortaleza. Pró-Fono R. Atual. Cient. v. 8, n. 2, p. 15-24, 1996.
RODRIGUES, R.; AZEVEDO, R.; \& BEHLAU, M. Considerações sobre voz profissional falada. In: MARCHESAN, I.; ZORZI; J.; \& GOMES, J. Tópicos de fonoaudiologia. v. 3. São Paulo: Lovise, 1996. p. 701-711.

RUSSELL, A.; OATES, J.; \& GREENWOOD, K. M. Prevalence of voice problems in teachers. J. Voice. v. 12, n. 4, p. 467-479, 1998.

SCALCO, M. A. G.; PIMENTEL, R. M.; \& PILZ, W. A saúde vocal do professor: levantamento junto às escolas particulares de Porto Alegre. Pró-Fono R. Atual. Cient. v. 8, n. 2, p. 25-31, 1996.

SIMÕES, M. Prevalência de disfonia e seus fatores associados em educadores de creche. 125f. 2001. Dissertação (Mestrado em Epidemiologia e Controle de Agravos à Saúde) - Faculdade de Saúde Pública, Universidade de São Paulo, São Paulo, 2001.

SMITH, E. et al. Voice problems among teachers: differences by gender and teaching characteristics. J. Voice. v. 12, n. 3, p. 328-334, 1998.

TENOR, A. C.; CYRINO, E. G.; \& GARCIA, V. L. Investigação da percepção vocal de professores de pré-escolas da rede municipal de ensino de Botucatu. Revista Salusvita, v. 18, n. 2, p. 107-116, 1999.

URRUTIKOETXEA, A.; ISPIZUA, A.; \& MATELLANES, F. Pathologie vocale chez les professeurs: une étude vídeo-laryngostroboscopique de 1046 professeurs. Rev. Laryngol. Otol. Rhinol. v. 116, n. 4, p. 255262, 1995.

VIOLA, I. C. et al. Voz do professor: levantamento das publicações brasileiras. Rev. Soc. Bras. Fonoaudiol., v. 5, n. 7, p. 36-47, 1999. 